Pierre Piron

Suppléant aux Codes Congolais, Législation de la République Démocratique du Congo 1960 - 1970, avec des notes de concordance et de doctrine et des commentaires extraits des travaux préparatoires, des circulaires et instructions officielles.

Brüssel 1970, 4 Bände, getrennt paginiert: 108, 291, 376, 103 S., Zusammen 1900 FB, Verlag Ferdinand Larcier, Brüssel

Kurz vor der Unabhängigkeit von Belgisch-Kongo (seit 27. 10. 71 Zaire) erschien die 8. Auflage der Ausgabe der geltenden Gesetzgebung von Pierre Piron und Jacques Devos (Codes et lois du Congo Belge, 3 Bände, Brüssel 1960). Das vorliegende Werk stellt dazu eine Ergänzung für die erste Dekade des unabhängigen Kongo dar (1960-1970), enthält also alle noch geltenden Vorschriften, die der selbständige Staat in diesen zehn Jahren erlassen hat, sowie zahlreiche Hinweise auf Literatur, Rechtsprechung und Verwaltung sowie auf aufgehobene Vorschriften. So ist z. B. nach der geltenden dritten Verfassung vom 24. Juni 1967 ein Abschnitt über die beiden früheren Verfassungen vom 19. Mai 1960 und 1. August $1964 \mathrm{zu}$ finden, in dem zwar nicht die Verfassungstexte, aber alle Materialhinweise und zusätzliche Vorschriften enthalten sind. (Bd. I, S. 13 ff.). $\mathrm{Da}$ die StA früher im BGB des Kongo geregelt war, wird das geltende StAG vom 18. September 1965 noch unter der Rubrik "Code civil“ abgedruckt, wo es ein Außenstehender allerdings nicht vermuten würde. Für solche Schwierigkeiten leistet der vierte Band (Register) gute Dienste; dort finden sich Sachregister und chronologische Register, sowohl des vorliegenden Werkes wie des Grundwerkes von 1960. Die übrigen drei Bände betreffen: Bürgerliches, Handels- und Strafrecht; Gerichte, Verfahren und Verwaltung; Wirtschafts- und Sozialrecht. Die allgemeinen völkerechtlichen Verträge sind im ersten Teil nach der Verfassung zu finden, die Verträge über besondere Rechtsmaterien sind am jeweiligen Ort klassifiziert.

Der Verfasser des Grundwerkes von 1960, der erster Präsident des Cour d'appel in Léopoldville (Kinshasa) wurde, veranstaltete auch dieses Ergänzungswerk, und zwar auf Initiative des Staatspräsidenten Mobutu.

Hellmuth Hecker

\title{
Peter Ranis
}

FIVE LATINAMERICAN NATIONS - A COMPARATIVE POLITICAL STUDY New York (The Macmillan Company), London (Collier-Macmillan Limited) 1971, $337 \mathrm{~S}$.

Das in den vergangenen Jahren stetig gewachsene Interesse an Lateinamerika hat sich auch in der Offentlichkeit nach und nach vom romantischen Flair des heißen Amazonas und den uns in der Form von Kriminalgrotesken erreichenden Nachrichten aus Uruguay insbesondere nach den Ereignissen in Peru und Chile auf die politische und wirtschaftliche Aktualität des Subkontinents verlagert. Eine Fülle von jüngeren Publikationen darf dafür als Indiz angesehen werden.

In der vorliegenden Arbeit nimmt sich Peter Ranis vor, die politische Entwicklung Lateinamerikas von der Unabhängigkeit bis zur Gegenwart in der Form eines übersichtlichen „Readers" beschreibend und interpretierend am Beispiel von Mexiko, Brasilien, Argentinien, Chile und Peru darzustellen. 
Eingeleitet wird die Studie mit einem allgemeinen Teil über den Modernisierungsprozeß in Lateinamerika im Zusammenhang mit der Veränderung des politischen Systems. Ein zweiter Teil faßt die politischen Systeme der präkolumbianischen und kolonialen Epoche einführend für die schwerpunktmäßige Behandlung der nachkolonialen Periode zusammen. Diese Periode wird in drei typisierten Phasen beschreibend dargestellt. Es folgen Darstellungen der „political culture“, der politischen Machtgruppen und ein interpretierendes Schlußkapitel über den Zusammenhang zwischen politischem, wirtschaftlichem und sozialem System.

Die naheliegende Frage nach der Vergleichbarkeit und Ubertragbarkeit der Ergebnișse wird nur am Rande gestreift; da im Bereich der Theorien und Kategoriensysteme, in denen Ranis seine Analyse ansiedelt, ein globales, begrifflich eindeutiges, logisch stringentes und empirisch gültiges theoretisches System nicht vorliegt, beschränkt sich sein Zugriff auf "case studies“, aus denen allgemeine Hypothesen über den Prozeß des politischen Wandels abzuleiten nicht ohne weiteres möglich erscheint. Insbesondere bleibt offen, wie die wirtschaftlichen Bedingungen, das politische System und die konkurrierenden Ideologien auch oder besonders in Abhängigkeit globaler Außenbeziehungen auf einem höheren Abstraktionsniveau als auf dem von isolierten „case studies“ ineinandergreifen; man vermißt eine Auseinandersetzung mit entsprechenden Aussagen aus dem Bereich der politischen Ókonomie, die sich Ranis auch dadurch versagt, daß er sich in Quellen und Zitaten fast ausschließlich auf Vertreter der angelsächsischen Politologie bezieht.

Die vorliegende Arbeit von Peter Ranis ist eine gute einführende Ergänzung für die neuere sozialwissenschaftliche Literatur über Lateinamerika, auch wenn man $\mathrm{ihm}$ in manchen Interpretationen und Prognosen nicht immer folgen möchte und obgleich manche Fragen offen bleiben, auf die Antworten suggeriert werden.

Manfred Wöhlcke

\section{RENGeling, Hans-WeRner}

\section{Privatvölkerrechtliche Verträge}

Zum Rechtscharakter eines Vertrages zwischen einem Staat und einer ausländischen Privatperson (oder einem nichtstaatlichen Verband)

Schriften zum Völkerrecht: Band 16

Duncker \& Humblot, Berlin 1971, 272 S., DM 49,60

Die Frage nach dem Rechtscharakter der zwischen Staaten und ausländischen Privatpersonen abgeschlossenen Verträge gehört zu den interessantesten und umstrittensten Themen des Völkerrechts. Das Phänomen dieser Verträge, die durch die Erweiterung des grenzüberschreitenden Wirtschaftsverkehrs an Zahl und Bedeutung ständig wachsen, stellt den Völkerrechtler vor dogmatisch schwierige Einordnungsprobleme.

Auch die vorliegende Arbeit, der die Dissertation des Verfassers zugrundeliegt, bemüht sich, einen Ausweg aus den in der traditionellen Lehre der Völkerrechtsfähigkeit wurzelnden Einordnungsproblemen aufzuweisen. Dazu verläßt der Verfasser die Bahnen des dualistischen Rechtsdenkens und bemüht sich, diese Verträge in einer Rechtssphäre $\mathrm{zwischen} \mathrm{dem} \mathrm{klassischen} \mathrm{Völkerrecht} \mathrm{und} \mathrm{dem} \mathrm{staatlichen}$ Recht anzusiedeln, indem er sie dem von ihm erfundenen Privatvölkerrecht unterstellt. 